\title{
SOME ADDITION THEOREMS OF GROUP THEORY WITH APPLICATIONS TO GRAPH THEORY
}

\author{
H. P. YAP
}

I. Introduction and definitions. Let $G$ be an additive group with nonempty subsets $S$ and $T$. Let $S \pm T=\{s \pm t ; s \in S, t \in T\}$, let $\bar{S}$ be the set complement of $S$ in $G$, and let $|S|$ be the cardinality of $S$. We abbreviate $\{f\}$, where $f \in G$ to $f$. If $S+S$ and $S$ have no element in common, then we say that $S$ is a sum-free set in $G$ or that $S$ is sum-free in $G$. If $S$ is a sum-free set in $G$ and if for every sum-free set $T$ in $G,|S| \geqq|T|$, then $S$ is said to be a maximal sum-free set in $G$. We denote by $\lambda(G)$ the cardinality of a maximal sum-free set in $G$. We say that $S$ is in arithmetic progression having difference $d$ if $S=\{s, s+d, \ldots, s+n d\}$ for some $s, d \in G$ and some integer $n \geqq 0$.

If $|G|$ is finite, then the following four cases are exhaustive and mutually exclusive.

Case 1. $|G|$ is even.

Case 2. $|G|$ is odd and $|G|$ has at least one prime factor congruent to 2 modulo 3.

Case 3. $|G|$ has no prime factor congruent to 2 modulo 3 but has 3 as a factor.

Case 4. Every prime factor of $|G|$ is congruent to 1 modulo 3 .

In $[\mathbf{2}]$, the following theorems for finite abelian groups $G$ were proved.

Theorem I.1. In Case 1 , let $|G|=2 m$. Then $\lambda(G)=m$ and if $S$ is a maximal sum-free set in $G, S=\bar{H}$ where $H$ is a subgroup of $G$.

Theorem I.2. In Case 2, let $|G|=p m$, where $p=3 k+2$ is the smallest prime factor congruent to 2 modulo 3 of $|G|$. Then

$$
\lambda(G)=(k+1) m .
$$

Moreover, if $S$ is a maximal sum-free set in $G$, then $S$ is a union of cosets of some subgroup $H$, of order $m$, of $G, S / H$ is in arithmetic progression, and $S \cup(S+S)=G$.

Theorem I.3. In Case $3, \lambda(G)=|G| / 3$. Moreover, if $S$ is a maximal sum-free set in $G$, then $S$ is a union of cosets of some subgroup $H$, of order $|G| / 3 m$, of $G$, where $m$ is an integer such that $3 m|| G \mid$, and one of the following holds:

(i) $|S+S|=2|S|-|H|$,

(ii) $|S+S|=2|S|$ and $S \cup(S+S)=G$.

Received February 9, 1970. 
Theorem I.4. In Case 4, we have

$$
\frac{1}{3}|G|\left(1-\frac{1}{m}\right) \leqq \lambda(G) \leqq \frac{1}{3}(|G|-1),
$$

where $m=\max \{n ; n$ is the order of $g, g \in G\}$.

It is clear that if $S$ is sum-free in $G$, then $(S-S) \cap S=\emptyset$. In this paper we answer the following questions. Suppose that $A^{*}$ is the collection of all subsets of cardinality $\lambda(G)$ of a finite abelian group $G$; what is the maximum cardinality, denoted by $i\left(G_{\lambda}\right)$, of subsets $M$ of $G$ such that $(M-M) \cap A=\emptyset$ for $A \in A^{*}$ ? Suppose that $M^{*}$ is the collection of all subsets $M$, of cardinality $i\left(G_{\lambda}\right)$, of $G$; what is the maximum cardinality of subsets $A$, denoted by $d\left(G_{\lambda}\right)$, of $G$ such that $(M-M) \cap A=\emptyset$ for $M \in M^{*}$ ?

We find that the results obtained here can be applied to the construction of certain classes of group-graphs satisfying some critical conditions. By a group-graph $\left\langle G, R_{A}\right\rangle$, we mean a graph with $G, R_{A}$ as follows: $G$ is an additive group with a non-empty subset $A$. The relation $R_{A} \subseteq(G, G)$ is the set of all ordered pairs $\left(g, g^{\prime}\right)$ such that $g, g^{\prime} \in G$ and $g-g^{\prime} \in A$.

Since $R_{A}$ depends only on $A$, we shall henceforth write $\langle G, A\rangle$ for $\left\langle G, R_{A}\right\rangle$.

A group-graph $\langle G, A\rangle$ can be interpreted as an ordinary graph as follows. Take each element $g$ of $G$ to be a vertex on the paper. Let $g, g^{\prime} \in G$. If $g-g^{\prime} \in A$ but $g^{\prime}-g \notin A$, then draw a directed line segment joining $g$ to $g^{\prime}$; if $g-g^{\prime} \in A$ and $g^{\prime}-g \in A$, then draw an undirected line segment joining $g$ and $g^{\prime}$; if $g-g^{\prime} \notin A$, and $g^{\prime}-g \notin A$, then no line segment is drawn joining $g$ and $g^{\prime}$.

It has been proved $[\mathbf{5} ; \mathbf{6}]$ that every group-graph is point-symmetric. (The definition of a group-graph in [5] is different from the definition given above, but it can be proved that for the undirected case the two definitions are equivalent.)

On the other hand, although it is not true that every finite point-symmetric graph is a group-graph (the Petersen graph is point-symmetric, but not a group-graph [9]), it is true that every point-symmetric graph with a prime number of vertices is a group-graph [7]. Thus, the results obtained in this paper have some significance in graph theory. In fact, if we are interested only in group-graphs, we can, by methods similar to that used here, enhance the results obtained in this paper.

The following is an example of a group-graph $\langle G, A\rangle$ :

$$
G=C_{4} \times C_{2}=\{(i, j) ; 0 \leqq i \leqq 3,0 \leqq j \leqq 1\}
$$

is the direct product of two cyclic groups $C_{4}$ of order 4 and $C_{2}$ of order 2;

$$
A=\{(0,1),(1,1),(2,1),(3,1)\} .
$$

Let $\langle G, A\rangle$ be a group-graph. Then $\langle G, A\rangle$ is of degree $m$ if and only if $|A|=m$. The order of $\langle G, A\rangle$ is the order of $G .\langle G, A\rangle$ is undirected if and only if $-A=A$ and $\langle G, A\rangle$ is loopless if and only if $0 \notin A$. 


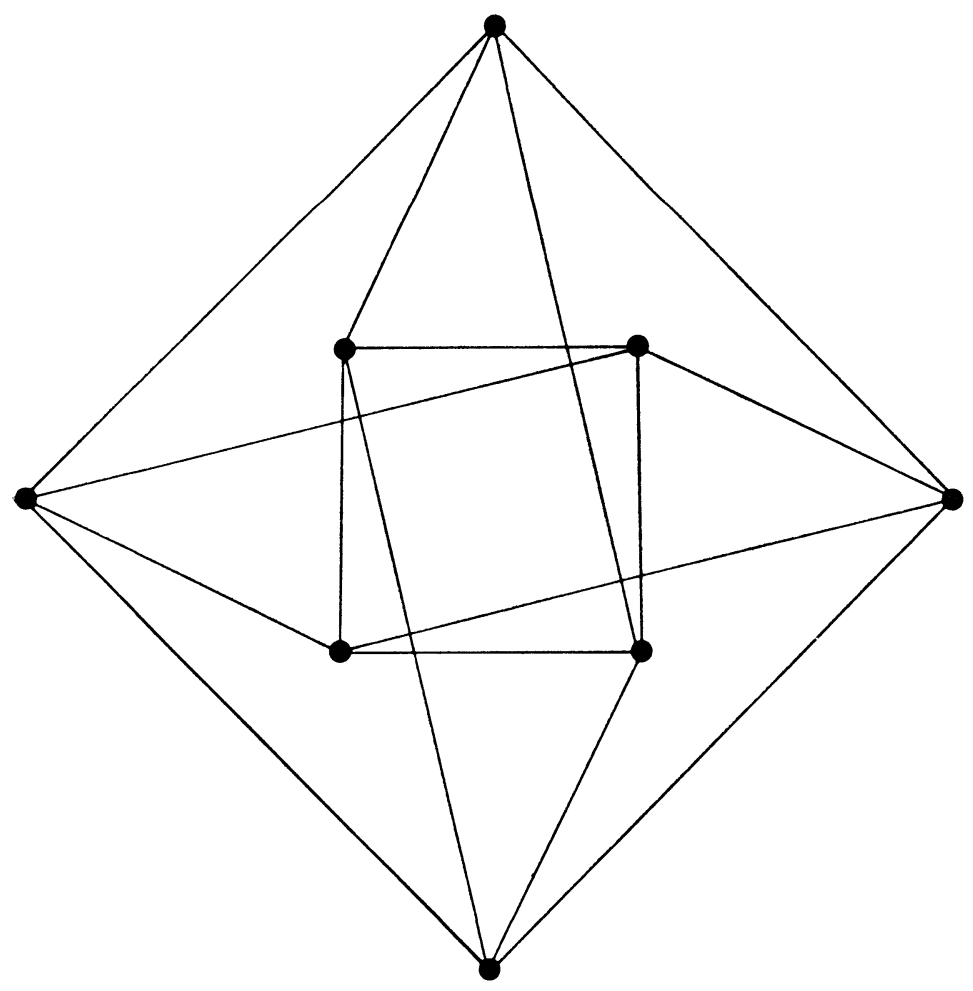

In the subsequent discussions, we shall make use of the following theorems [3, Chapter I].

Theorem I.5 (Mann). Let $G$ be a group with subsets $A$ and $B$. Then either $A+B=G$ or $|G| \geqq|A|+|B|$.

Theorem I.6 (Cauchy-Davenport). If $A$ and $B$ are subsets of a group $G$ of prime order $p$, then $A+B=G$ or $|A+B| \geqq|A|+|B|-1$.

THEOREM I.7 (Vosper). Let $G$ be the additive group of residues modulo a prime p. Let $A$ and $B$ be subsets of $G$ and $C=A+B$. Then either $|C| \geqq|A|+$ $|B|$ or one of the following holds: (i) $C=G$; (ii) $|C|=p-1$ and $\bar{B}=f-A$, where $f=\bar{C}$; (iii) $A$ and $B$ are in arithmetic progression and have the same difference; (iv) $|A|=1$ or $|B|=1$.

Theorem I.8 (Kneser). Let $G$ be an abelian group with finite subsets $A$ and $B$. Then there exists a subgroup $H$ of $G$ such that

$$
A+B+H=A+B \quad \text { and } \quad|A+B| \geqq|A+H|+|B+H|-|H| \text {. }
$$

II. The number $i\left(G_{\lambda}\right)$ of a finite abelian group $G$. Throughout this section, unless otherwise stated, $G$ stands for a finite abelian group. Let $A^{*}$ be the collection of all subsets of cardinality $\lambda(G)$ of $G$. Let $A \in A^{*}$. If $M$ is 
a subset of $G$ such that $(M-M) \cap A=\emptyset$ and $|M| \geqq\left|M^{\prime}\right|$ for any subset $M^{\prime}$ of $G$ satisfying $\left(M^{\prime}-M^{\prime}\right) \cap A=\emptyset$, then we say that $M$ is a maximal subset of $G$ satisfying $(M-M) \cap A=\emptyset$. It is clear that $M$ depends on $A$. We denote by $\mu(A)$ the cardinality of $M$ if $M$ is a maximal subset of $G$ satisfying $(M-M) \cap A=\emptyset$. We define

$$
i\left(G_{\lambda}\right)=\max _{A \in A^{*}} \mu(A) .
$$

It is clear that $i\left(G_{\lambda}\right)$ is the maximum coefficient of internal stability of group-graphs $\langle G, A\rangle$ of degree $\lambda(G)[\mathbf{1}$, p. 35].

If $A \in A^{*}$ and if $M$ is a maximal subset of $G$ satisfying $(M-M) \cap A=\emptyset$, $|M|=i\left(G_{\lambda}\right)$, then $(A, M)$ is said to be a pair of critical subsets of $G$ satisfying the $\lambda$-conditions.

Let $S$ be a maximal sum-free set in $G$, then $(S-S) \cap S=\emptyset$. Thus we have the following result.

Lemma II.1. $i\left(G_{\lambda}\right) \geqq \lambda(G)$.

Now, suppose that $(A, M)$ is a pair of critical subsets of $G$ satisfying the $\lambda$-conditions, then by Theorem I.8 there exists a subgroup $H$ of $G$ such that $M-M+H=M-M$ and $|M-M| \geqq|M+H|+|-M+H|-|H|$. From $M-M=M-M+H=(M+H)-(M+H)$, we have

$$
((M+H)-(M+H)) \cap A=\emptyset .
$$

Since $|M+H| \geqq|M|$, therefore, by the hypothesis, $M+H=M$. Hence we have the following result.

Lemma II.2. $(A, M+H)$ is also a pair of critical subsets of $G$ satisfying the $\lambda$-conditions and thus $M$ is a union of cosets of $H$.

From this lemma, we have

$$
|G|-\lambda(G) \geqq|M-M| \geqq 2|M|-|H|,
$$

and thus

$$
|M| \leqq\left[\frac{1}{2} \frac{|G|-\lambda(G)}{|H|}+\frac{1}{2}\right]|H|,
$$

where $[x]$ denotes the integer part of $x$. Hence

$$
i\left(G_{\lambda}\right) \leqq \max _{a|| G \mid}\left[\frac{1}{2} \frac{|G|-\lambda(G)}{d}+\frac{1}{2}\right] d .
$$

Now we prove the following.

Lemma II.3. Let $G$ be a group (not necessarily abelian). If $A$ and $B$ are finite subsets of $G$ satisfying $|A+B|=|A| \geqq|B|$, then $A$ is a union of cosets of a subgroup $H$ of $G$ such that $B-B \subseteq H$. 
Proof. Let $H$ be the set of all $h \in G$ such that $A+h=A$. It is clear that $H$ is a subgroup of $G$ and thus $A$ is a union of right cosets of $H$.

Now, from $|A+B|=|A|$, it follows that $A+b=A+b^{\prime}$ for every $b, b^{\prime} \in B$ and thus $A+b-b^{\prime}=A$ for every $b, b^{\prime} \in B$.

Hence $A+B-B=A$ and therefore $B-B \subseteq H$.

(We note that this lemma generalizes [2, Theorem 7].)

Theorem II.1. In Case 1, let $|G|=2 m$. Then

$$
i\left(G_{\lambda}\right)=m=\lambda(G) .
$$

Moreover, if $(A, M)$ is a pair of critical subsets of $G$ satisfying the $\lambda$-conditions, then $M-M$ is a subgroup of $G$ and $A=\overline{M-M}$ is a maximal sum-free set in $G$.

Proof. By Theorem I.5, $i\left(G_{\lambda}\right) \ngtr m$ and thus by Lemma I.1,

$$
i\left(G_{\lambda}\right)=m=\lambda(G) \text {. }
$$

Now, if $(A, M)$ is a pair of critical subsets of $G$ satisfying the $\lambda$-conditions, then $|M|=m$ and since $(M-M) \cap A=\emptyset,|A|=m$, therefore

$$
m \geqq|M-M| \geqq|M|=m
$$

and thus $|M-M|=|M|$. Now applying Lemma II.3, $M-M$ is a subgroup of $G$ and $A=\overline{M-M}$ is a maximal sum-free set in $G$.

The proof of Theorem II.1 is now complete.

We note that in the above theorem, $-A=A, 0 \notin A$, and thus by $[\mathbf{1}$, p. 46, Theorem 2], it follows that $M$ is a kernel [1, p. 45] of $\langle G, A\rangle$.

Theorem II.2. In Case 2, let $|G|=$ pm, where $p=3 k+2$ is the smallest prime factor congruent to 2 modulo 3 of $|G|$. Then

$$
i\left(G_{\lambda}\right)=(k+1) m=\lambda(G) .
$$

Moreover, if $(A, M)$ is a pair of critical subsets of $G$ satisfying the $\lambda$-conditions, then $M$ is a union of cosets of a subgroup $H$, of order $m$, of $G, M / H$ is in arithmetic progression, and $A=\overline{M-M}$ is a maximal sum-free set in $G$.

Proof. From (1), we have $(2 k+1) m \geqq|M-M| \geqq 2(k+1) m-|H|$. Thus $|H| \geqq m$. We shall first prove that $|H|=m$.

Suppose that $|H|>m$. Let

$$
|H|=p m_{1}, \quad m=m_{1} m_{2} ;
$$

then $p>m_{2}>1$. Thus

$$
|M| \geqq\left(\frac{(k+1) m}{p m_{1}}\right] p m_{1},
$$

where $(x]$ denotes the smallest integer greater than or equal to $x$. 
Case (i). When $m_{2}=3 m_{3}$,

$$
\left(\frac{(k+1) m}{p m_{1}}\right]=\left(\frac{(3 k+2) m_{3} m_{1}+m_{3} m_{1}}{p m_{1}}\right]=m_{3}+1 .
$$

Hence $|M-M| \geqq 2\left(m_{3}+1\right) p m_{1}-p m_{1}=(2 k+1) m+m / 3+p m_{1}$, which is impossible.

Case (ii). When $m_{2}=3 m_{3}+1$,

$$
\left(\frac{(k+1) m}{p m_{1}}\right]=\left(\frac{(3 k+2) m_{3} m_{1}+\left(m_{3}+(k+1)\right) m_{1}}{p m_{1}}\right] \text {. }
$$

But $p / 3>m_{3}+1 / 3, p / 3=k+2 / 3$, therefore $2 p / 3>m_{3}+(k+1)$. Hence

$$
\left(\frac{(k+1) m}{p m_{1}}\right]=m_{3}+1
$$

and $|M-M| \geqq 2\left(m_{3}+1\right) p m_{1}-p m_{1}=(2 k+1) m+m / 3+(k+2 / 3) m_{1}$, which is impossible.

We note that $m_{2} \neq 3 m_{3}+2$ since $p$ is the smallest prime factor congruent to 2 modulo 3 of $|G|$. Hence $|H|=m$ and from (1), again, we have

$$
(2 k+1) m \geqq|M-M| \geqq 2|M|-m \geqq(2 k+1) m \text {. }
$$

It follows that $|M|=(k+1) m$ and $|M-M|=2|M|-|H|$. Thus

$$
|(M-M) / H|=2|M / H|-1 \text {. }
$$

Applying Theorem I.7, we know that $M / H$, being a subset of the quotient group $G / H$, or prime order $p$, is in arithmetic progression. Let

$$
M / H=\{a+j d ; j=0,1, \ldots, k\}, \quad a, d(\neq 0) \in G / H .
$$

Then $\overline{((M-M) / H)}=\{(k+1) d,(k+2) d, \ldots,(2 k+1) d\}$ is sum-free in $G$ and thus

$$
\begin{aligned}
& A=(H+(k+1) d) \cup(H+(k+2) d) \cup \ldots \cup(H+(2 k+1) d), \\
& \text { is a maximal sum-free set in } G .
\end{aligned}
$$

The proof of Theorem II.2 is now complete.

We note that $M$ appearing in the above theorem is a kernel of $\langle G, A\rangle$. This follows from [8, Lemma 1$]$ and $[\mathbf{1}$, p. 46, Theorem 2].

Theorem II.3. In Case 3 , let $|G|=3^{n} m$, where $(3, m)=1$. Then

$$
i\left(G_{\lambda}\right)=3^{n-1} m=\lambda(G) .
$$

Moreover, if $(A, M)$ is a pair of critical subsets of $G$ satisfying the $\lambda$-conditions, then one of the following holds:

(i) $|M-M|=2|M|-|H|$,

(ii) $|M-M|=2|M|$ and $A \cup(M-M)=G$. 
Proof. If

then

$$
\left[\frac{1}{2} \frac{|G|-\lambda(G)}{d}+\frac{1}{2}\right] d>3^{n-1} m, \quad d|| G \mid,
$$

$$
\left[\frac{3^{n-1} m}{d}+\frac{1}{2}\right] d>3^{n-1} m, \quad d|| G \mid .
$$

Thus $d=3^{n} m_{1}, 1 \leqq m_{1}<m, m=m_{1} m_{2}, m_{2}=3 q+1$. Therefore $q d>3^{n-1} m$, which is impossible. Hence $i\left(G_{\lambda}\right)=3^{n-1} m=\lambda(G)$.

From $2|M| \geqq|M-M| \geqq 2|M|-|H|$ and Lemma II.2, we have

$$
2|M / H| \geqq|(M-M) / H| \geqq 2|M / H|-1 \text {. }
$$

Thus, one of the following holds:

(i) $|M-M|=2|M|-|H|$,

(ii) $|M-M|=2|M|$ and thus $A=\overline{M-M}, A \cup(M-M)=G$.

The proof of Theorem II.3 is now complete.

We remark that in the above theorem, if (ii) holds, then, since $-A=A$ and $0 \notin A$, by [1, p. 46, Theorem 2], $M$ is a kernel of $\langle G, A\rangle$.

TheOREM II.4. In Case 4, suppose that $G$ is the additive group of residue classes modulo $|G|$; then

$$
i\left(G_{\lambda}\right)=(|G|+2) / 3 .
$$

Moreover, if $(A, M)$ is a pair of critical subsets of $G$ satisfying the $\lambda$-conditions, then $|M-M|=2|M|-1$ and $A \cup(M-M)=G$.

Proof. Suppose that $|G|=3 k+1$. Let

$$
M=\{a, a+d, \ldots, a+k d\}, \quad(d,|G|)=1 .
$$

Then

and

$$
M-M=\{0, \pm d, \pm 2 d, \ldots, \pm k d\}
$$

$$
A=\overline{M-M}=\{(k+1) d,(k+2) d, \ldots, 2 k d\} .
$$

Hence $i\left(G_{\lambda}\right) \geqq k+1$.

On the other hand, from (1), we have $2 k+1 \geqq|M-M| \geqq 2|M|-|H|$. If $|M|>k+1$, then $|H| \geqq 3$. Let

$$
|H|=3 h+1, \quad|G| /|H|=3 h^{\prime}+1 .
$$

Then $3 k+1=(3 h+1)\left(3 h^{\prime}+1\right)$ and thus $k=h^{\prime}|H|+h$. Hence

$$
|M| \geqq\left(\frac{k+2}{|H|}\right]|H| \geqq\left(h^{\prime}+1\right)|H|,
$$

from which it follows that

$$
\begin{array}{r}
2 h^{\prime}|H|+2 h+1=2 k+1 \geqq|M-M| \geqq 2\left(h^{\prime}+1\right)|H|-|H| \\
=\left(2 h^{\prime}+1\right)|H|,
\end{array}
$$

which is impossible. Hence $i\left(G_{\lambda}\right)=k+1=(|G|+2) / 3$. 
Next, if $(A, M)$ is a pair of critical subsets of $G$ satisfying the $\lambda$-conditions, then from (1), we have

$$
2 k+1 \geqq|M-M| \geqq 2|M+H|-|H| .
$$

By Lemma II.2, $|H||| M \mid=k+1$; therefore,

$$
|H|(|M|,|G|)=(k+1,3 k+1)=1 .
$$

Hence $|H|=1$ and thus

$$
2 k+1 \geqq|M-M| \geqq 2|M|-1=2 k+1,
$$

from which it follows that $|M-M|=2|M|-1$ and $A \cup(M-M)=G$.

The proof of Theorem II.4 is now complete.

We remark that in the above theorem, since $-A=A$ and $0 \notin A$, by $[\mathbf{1}$, p. 46 , Theorem 2], $M$ is a kernel of $\langle G, A\rangle$.

III. The number $d\left(G_{\lambda}\right)$ of a finite abelian group $G$. Throughout this section, $G$ stands for a finite abelian group. Let $M^{*}$ be the collection of all subsets $M$, of cardinality $i\left(G_{\lambda}\right)$, of $G$. Define

$$
d\left(G_{\lambda}\right)=|G|-\min _{M \in M^{*}}|M-M| .
$$

If $A \subseteq G$ is such that $(M-M) \cap A=\emptyset, M \in M^{*},|A|=d\left(G_{\lambda}\right)$, then $\langle G, A\rangle$ is a group-graph with maximum degree such that the coefficient of internal stability is $i\left(G_{\lambda}\right)$.

If $M \in M^{*}$ is such that $|M-M|=|G|-d\left(G_{\lambda}\right)$, then $M$ is said to satisfy the $d$-conditions.

From (1), we have the following result.

LEMMA III.1. $d\left(G_{\lambda}\right) \geqq \lambda(G)$.

Now, if $M$ satisfies the $d$-conditions, let $A=\overline{M-M}$; then

$$
(A+M) \cap M=\emptyset .
$$

By Theorem I.8, there exists a subgroup $K$ of $G$ such that

$A+M+K=A+M \quad$ and $\quad|A+M| \geqq|A+K|+|M+K|-|K|$,

from which it follows that

$$
|G|-i\left(G_{\lambda}\right) \geqq|A+M| \geqq|A+K|+|M+K|-|K| .
$$

It is clear that the converse of Theorem II.1 is also true. In fact, we have the following result.

Theorem III.1. In Case 1 , let $|G|=2 m$. Then

$$
d\left(G_{\lambda}\right)=m=\lambda(G) .
$$


Moreover, if $M$ satisfies the d-conditions, then $A=\overline{M-M}$ is a maximal sum-free set in $G$.

Now we prove that the converse of Theorem II.2 is also true.

Theorem III.2. In Case 2, let $|G|=p m$, where $p=3 k+2$ is the smallest prime factor congruent to 2 modulo 3 of $|G|$. Then

$$
d\left(G_{\lambda}\right)=(k+1) m=\lambda(G) .
$$

Moreover, if $M$ satisfies the d-conditions, then there exists a subgroup $K$, of order $m$, of $G$, such that $A=\overline{M-M}$ and $M$ are unions of cosets of $K$. Also $M / K$ and $A / K$ are in arithmetic progression and have the same difference.

Proof. From (3), we have

$$
(2 k+1) m \geqq|A+M| \geqq|A|+|M|-|K| \geqq 2(k+1) m-|K| .
$$

Thus $|K| \geqq m$.

Using a method similar to that in the proof of Theorem II.2, we can show that $|K|>m$. Thus $|K|=m$ and $|A+K|=(k+1) m=|A|, \quad|M+K|=$ $(k+1) m=|M|$ and therefore both $A$ and $M$ are unions of cosets of $K$. Hence

$$
|(A+M) / K|=|A / K|+|M / K|-1
$$

and by Theorem I.7, we know that $A / K$ and $M / K$ are in arithmetic progression and have the same difference.

Finally, by the same method used in the proof of Theorem II.2, we can show that $A=\overline{M-M}$ is a maximal sum-free set in $G$.

Remarks. In Case 2, by using Theorems II.2 and III.2 we see that $(A, M)$ is a pair of critical subsets of $G$ satisfying the $\lambda$-conditions if and only if $M$ is a subset of $G$ satisfying the $d$-conditions and $A=\overline{M-M}$ is a maximal sum-free set in $G$. Thus, in order to construct a group-graph $\langle G, A\rangle$ of degree $\lambda(G)$ with coefficient of internal stability $i\left(G_{\lambda}\right)$, we first find a subgroup $H$, of order $m$, of $G$; then by Theorem II.2,

$$
A=H+\{(k+1) d,(k+2) d, \ldots,(2 k+1) d\},
$$

where $d \in G, d \notin H$. Also, by Theorem III.2,

$$
M=H+\{a+j d ; j=0,1, \ldots, k\}
$$

for any $a \in G$.

For a fixed $d \in G, d \notin H$, let

$$
\begin{gathered}
M=H+\{a+j d ; j=0,1, \ldots, k\}, \\
M_{1}=H+\left\{a_{1}+j d ; j=0,1, \ldots, k\right\} .
\end{gathered}
$$

It is clear that if $a_{1} \notin a+H$, then $M_{1} \neq M$. Let

$$
M^{\prime}=H+\left\{a^{\prime}+j d^{\prime} ; j=0,1, \ldots, k\right\}, \quad d^{\prime} \in G, d^{\prime} \notin H .
$$


By [4, p. 276, Lemma 1], if $d^{\prime} \notin d+H$, then $M^{\prime} \neq M$. Thus, for a fixed maximal sum-free set $A$ in $G$, there are altogether $p(p-1) / 2$ distinct kernels of $\langle G, A\rangle$.

Theorem III.3. In Case 3, we have

$$
d\left(G_{\lambda}\right)=2|G| / 3=2 \lambda(G) .
$$

Moreover, if $M$ satisfies the $d$-conditions, then $K=M-M$ is a subgroup, of order $|G| / 3$, of $G$.

Proof. Let $H$ be a subgroup, of order $|G| / 3$, of $G$. Let $N$ be a coset of $H$. Then $|G|-|N-N|=2|G| / 3$. Thus

$$
d\left(G_{\lambda}\right) \geqq 2|G| / 3 .
$$

Now, let $M$ be a subset of $G$ satisfying the $d$-conditions. Then

$$
|\overline{M-M}|=|G|-|M-M| \leqq|G|-|M| \leqq|G|-\lambda(G)=2|G| / 3 .
$$

Hence $|A|=|\overline{M-M}|=2|G| / 3$ and thus $d\left(G_{\lambda}\right)=2|G| / 3$.

Next, from $2|G| / 3 \geqq|A+M| \geqq|A+K|+|M+K|-|K|$, it follows that $|K| \geqq|G| / 3$ and thus $|K|=|G| / 3$. Therefore $M$ is a coset of $K$ and thus $M-M=K$.

Theorem III.4. In Case 4, suppose that $G$ is cyclic; then

$$
d\left(G_{\lambda}\right)=(|G|-1) / 3=\lambda(G) .
$$

Moreover, if $M$ is a subset of $G$ satisfying the d-conditions, then $A=\overline{M-M}$ is such that $|A+M|=|A|+|M|-1, M \cup(A+M)=G$.

Proof. Let $M$ be a subset of $G$ satisfying the $d$-conditions. Let $|G|=3 k+1$. Then from (3), we have

$$
2 k \geqq|A+M| \geqq|A+K|+|M+K|-|K| .
$$

If $|A|>k$, then $2 k \geqq|A+M| \geqq(k+1)+(k+1)-|K|$, from which it follows that $|K| \geqq 3$.

Suppose that $|K|=3 r+1,|G| /|K|=3 s+1$. Then

$$
3 k+1=(3 r+1)(3 s+1)
$$

and thus $k=s|K|+r$. Hence

$$
|A+K| \geqq\left(\frac{k+1}{|K|}\right]|K|=(s+1)|K| \quad \text { and } \quad|M+K| \geqq(s+1)|K| \text {. }
$$

Thus $2 k \geqq|A+M| \geqq 2 s|K|+|K|$, which is impossible.

Hence $d\left(G_{\lambda}\right)=(|G|-1) / 3=\lambda(G)$.

Finally, it can be shown that, in the above inequality, $|K|=1$. Therefore $2 k \geqq|A+M| \geqq|A|+|M|-1=2 k$ and thus

$$
|A+M|=|A|+|M|-1, \quad M \cup(A+M)=G \text {. }
$$

Acknowledgement. I am grateful to Professor P. H. Diananda for helpful discussions. 


\section{REFERENCES}

1. C. Berge, Theory of graphs and its applications (Methuen, London, 1962).

2. P. H. Diananda and H. P. Yap, Maximal sum-free sets of elements of finite groups, Proc. Japan Acad. 45 (1969), 1-5.

3. H. B. Mann, Addition theorems (Interscience, New York, 1965).

4. H. B. Mann and J. E. Olson, Sums of sets in the elementary abelian group of type $(p, p)$, J. Combinatorial Theory 2 (1967), 275-284.

5. G. Sabidussi, On a class of fix-point-free graphs, Proc. Amer. Math. Soc. 9 (1958), 800-804.

6. H. H. Teh and H. P. Yap, Some construction problems of homogeneous graphs, Bull. Math. Soc. Nanyang Univ. 1964, 164-196.

7. J. Turner, Point-symmetric graphs with a prime number of points, J. Combinatorial Theory 3 (1967), 136-145.

8. H. P. Yap, The number of maximal sum-free sets in $C_{p}$, Nanta Math. 2 (1968), 68-71.

9. - A class of point-symmetric graphs, Nanta Math. 3 (1969), 100-109.

University of Singapore,

Singapore 